\title{
AAV vector distribution in the mouse respiratory tract following four different methods of administration
}

Lisa A. Santry ${ }^{\dagger}$, Joelle C. Ingrao ${ }^{\dagger}$, Darrick L. Yu, Jondavid G. de Jong, Laura P. van Lieshout, Geoffrey A. Wood and Sarah K. Wootton ${ }^{*}$

\begin{abstract}
Background: Targeted delivery of gene therapy vectors to the mouse respiratory tract is often performed via intranasal or intratracheal administration; however, there can be a great deal of variability between these methods, which could potentially influence experimental results. Improving the accuracy and precision of lung delivery will not only reduce the number of animals required to detect statistically significant differences, but may reduce the variability of studies from different laboratories.

Results: Here we evaluated three different methods of adeno-associated virus (AAV) vector administration to the respiratory tract in mice (intranasal, intubation, and intratracheal injection) and discuss the advantages, challenges, and shortcomings of each. We also present a modified-intranasal delivery technique that is superior to passive administration of vector into the nares of anesthetized supine animals. Transgene expression was consistently visible in the nasal cavity, trachea, and proximal to middle aspect of all lung lobes for all four methods, whereas transgene expression was consistently observed in the most distal aspect of lung lobes only with the intubation and intratracheal injection techniques. AAV vector genome copy numbers in the lung were approximately four-fold lower in mice that received vector via intranasal administration in comparison to the other three methods of vector delivery. The modified intranasal, intubation and intratracheal injection methods of vector administration did not yield statistical differences in AAV vector genome copy numbers in the lung. With regard to reproducibility of vector distribution within and between animals, the modified-intranasal technique was superior.
\end{abstract}

Conclusion: Our results show that mode of AAV vector administration to the murine respiratory tract should be selected based on desired target site and skill of the researcher, and that appropriate technique selection may greatly influence experimental outcomes.

Keywords: Adeno-associated virus, Vector instillation, Transgene expression, Intratracheal, Intranasal, Intubation, Respiratory tract, Respiratory epithelium

\section{Background}

Gene therapy is currently being evaluated for a wide range of acute and chronic lung diseases including those caused by single gene defects such as cystic fibrosis, alpha-1 antitrypsin deficiency and surfactant protein B deficiency [1]. While the lung provides a natural route of entry into the host and can be accessed in a

\footnotetext{
* Correspondence: kwootton@uoguelph.ca

${ }^{\dagger}$ Equal contributors

Department of Pathobiology, University of Guelph, Guelph, Ontario N1G 2W1, Canada
}

(c) The Author(s). 2017 Open Access This article is distributed under the terms of the Creative Commons Attribution 4.0 International License (http://creativecommons.org/licenses/by/4.0/ which permits unrestricted use, distribution, and reproduction in any medium, provided you give appropriate credit to the original author(s) and the source, provide a link to the Creative Commons license, and indicate if changes were made. The Creative Commons Public Domain Dedication waiver (http://creativecommons.org/publicdomain/zero/1.0/) applies to the data made available in this article, unless otherwise stated.

comparatively easy and noninvasive manner, methods for consistent and efficient delivery of viral vectors to the respiratory tract are required. There have been a number of studies evaluating variables such as volume of inoculum, effect of diluent, nature of anesthesia (inhaled vs. injected), and position of mouse [2, 3] and how these affect intranasal delivery of therapeutic substances in mouse models, but a comparison of different delivery methods using viral vectors has not been done. Targeted delivery of viral vectors to the mouse respiratory tract can be performed by a variety of different methods, 
some of which require extensive skill and training. In this report, four different methods of delivering viral vectors to the mouse respiratory tract were evaluated for ease and consistency, vector distribution, and invasiveness of the procedure with the aim of identifying the most effective and consistent method of delivery requiring minimal skill development.

For this study, a lung-tropic AAV serotype 6 vector [4] expressing the human placental alkaline phosphatase (hPLAP) reporter gene was administered to the respiratory tract of C57BL/6 mice by intranasal, modified intranasal, endotracheal intubation, or intratracheal injection (Fig. 1) and subsequently evaluated for transduction efficiency. A total of $1 \times 10^{11}$ vector genomes $(\mathrm{vg})$ were delivered in a fixed volume of $80 \mu \mathrm{l}$ (delivered either in $2 \times 40 \mu \mathrm{l}$ doses as in the case of intranasal and modified intranasal administration or one dose of $80 \mu \mathrm{l}$ as in the case of intubation and intratracheal administration) using isoflurane as the anesthetic.

Here we show that the modified-intranasal technique resulted in a similar and consistent distribution of vector to the upper and lower respiratory tract, including all lung lobes, in all mice examined. While the intratracheal and intubation methods resulted in somewhat inconsistent distribution of vector, particularly to one lung lobe, the efficiency of lung gene delivery was similar to that of the modified intranasal technique. However, only the intubation method resulted in consistent vector transduction of the distal most aspect of the lung. Intranasal delivery efficiently targeted vector to the upper respiratory tract and as expected, led to robust transduction of the nasal epithelium with significantly less vector transduction in the lung relative to the other three methods of vector administration. In conclusion, the modified-intranasal technique proved to be the most efficient and consistent method of vector delivery requiring the least amount of skill. Use of the modifiedintranasal technique for delivery of viral vectors to the mouse respiratory tract may improve the accuracy and precision of lung delivery, which may reduce variability between studies from different laboratories and can have animal welfare implications by reducing the number of animals required for detecting statistically significant differences.

\section{Results}

Gross analysis of transgene expression

Grossly, hPLAP transgene expression was visible in all nasal cavities, trachea, and the proximal to middle
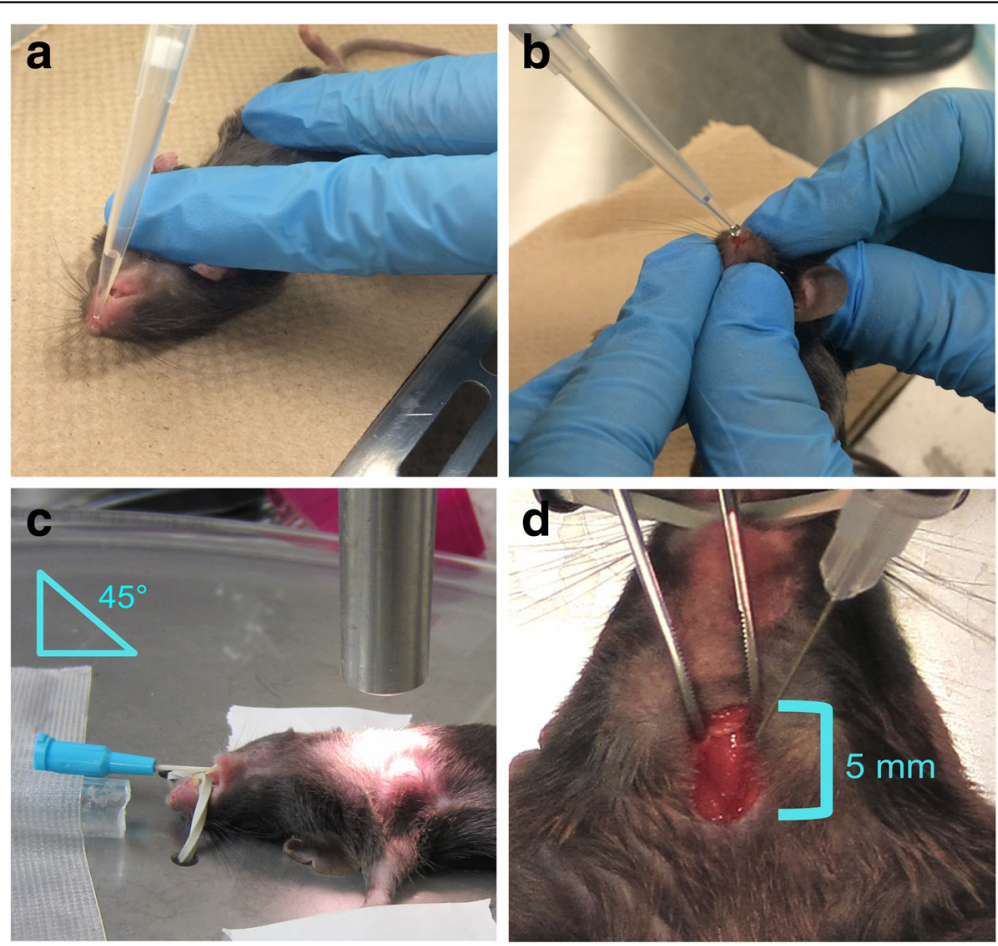

Fig. 1 Overview of the four different delivery methods used to administer viral vector to the mouse respiratory tract. a Intranasal technique. Mice were anesthetized with isoflurane and the viral vector administered drop wise over the nares allowing for passive inhalation of the substance. $\mathbf{b}$ Modifiedintranasal technique. Mice were anesthetized with isoflurane and the viral vector administered drop wise over the nares. The mouth is covered during administration to promote inhalation through the nose and subsequent distribution of the vector throughout the lower respiratory tract as well as to prevent swallowing of the administered substance. $\mathbf{c}$ Intubation technique. Mice were anesthetized with isoflurane in oxygen and a 22-gauge catheter placed into the trachea. The viral vector was administered to the airways through the catheter. $\mathbf{d}$ Intratracheal injection technique. Mice were anesthetized with isoflurane and the viral vector injected directly into the trachea following surgical visualization 
aspect of all lung lobes (Figs. 2, 3, 4, 5) for all four methods of vector administration. The intranasal technique resulted in staining of the proximal aspect of the lower respiratory tract with little to no staining of mid to distal aspects of lung lobes, respectively (Fig. 2). The modified-intranasal technique resulted in hPLAP staining in the proximal to mid-distal aspect of the lower respiratory tract, with no staining of the most distal aspects of lung lobes (Fig. 3). The intubation (Fig. 4) and intratracheal injection (Fig. 5) techniques resulted in hPLAP staining of the lower respiratory tract, up to and including distal aspects of lung lobes. For the modifiedintranasal technique, hPLAP expression was consistent between locations and between animals, whereas the intubation method resulted in markedly less hPLAP expression observed grossly in the middle lung lobe of half of the animals (Fig. 4c and d, lung lobe \#5). In one of four animals subjected to intratracheal administration of vector, hPLAP staining was restricted to the proximal aspect of the lower respiratory tract (Fig. 5d, lung lobe \#5). While more robust hPLAP expression was observed grossly in the nasal cavity following intranasal (Fig. 2) and modified intranasal delivery of vector (Fig. 3), both the intubation and intratracheal delivery methods resulted in varying degrees of hPLAP expression in the nasal cavity (Figs. 4-5).

\section{Histological analysis of AAV vector distribution in the mouse lung}

Efficiency of vector distribution in the lung at 21 days post-delivery of $1 \times 10^{11} \mathrm{vg}$ of an AAV6 vector expressing hPLAP by the intranasal, modified intranasal, intratracheal and intubation methods was evaluated histologically (Fig. 6 and Additional file 1 Figure S1, Additional file 2 Figure S2, Additional file 3 Figure S3, Additional file 4 Figure S4, Additional file 5 Figure S5,). Representative sections of paraffin-embedded lung tissue subjected to staining for hPLAP expression are shown in Figs. 6a-h. Histologically, AP staining was observed in bronchiole, bronchiolar, and alveolar epithelial cells in all mice for all techniques (Fig. 6a-h). Consistent with gross observations, a considerable amount of AP staining was observed in distal alveolar epithelial cells with the intubation (Fig. 6e and f) and intratracheal injection (Fig. $6 \mathrm{~g}$ and $\mathrm{h}$ ) techniques. In addition, AP expression was predominantly detected within the proximal airway following the modified intranasal technique. For all methods, AP expression was consistent between locations and between animals.

\section{Quantification of vector genome copy number in lungs}

We further evaluated the efficiency of AAV6 vector transduction via the four different delivery methods by

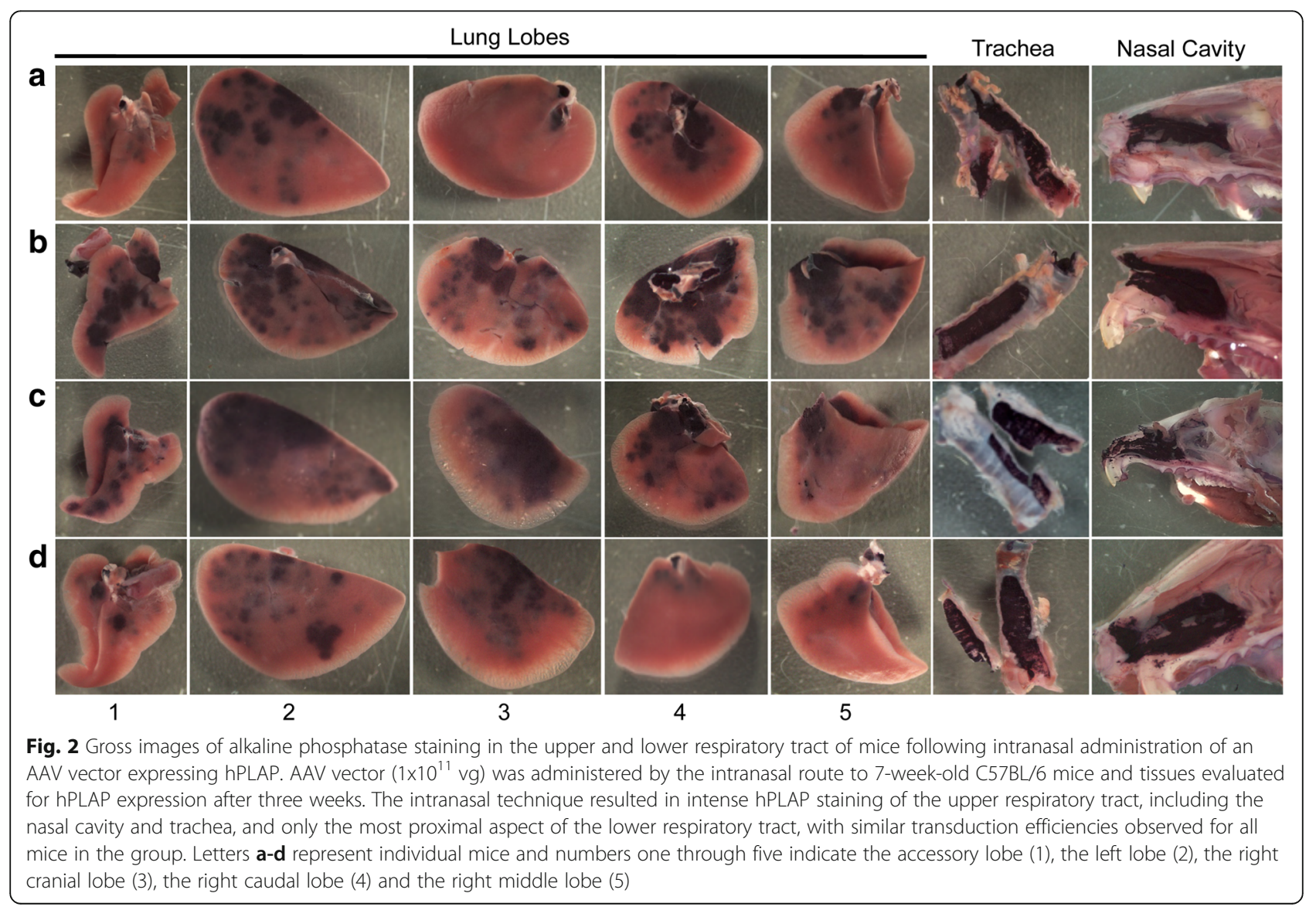




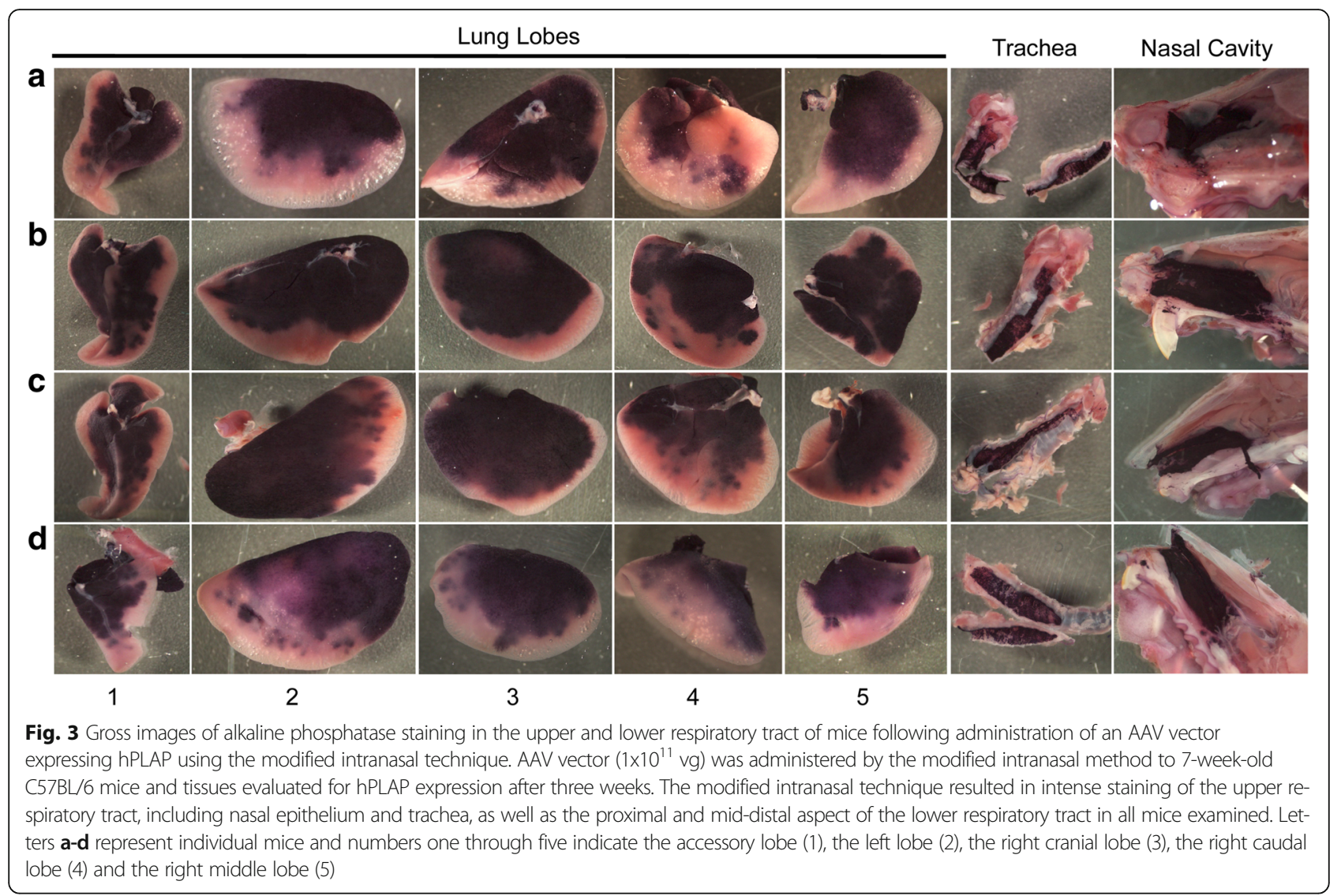

quantifying the number of vector genomes in the lungs of each of the experimentally infected mice at 21 days post-transduction. Vector copy number, which corresponds to viral genomes per ng of genomic DNA, was quantified using a well-established Taqman qPCR assay [5]. As shown in Fig. 7, the mean copy number of AAV vectors in the lung after intranasal delivery was approximately four-fold lower in comparison to the other three methods of vector delivery. The other three methods of vector delivery did not yield statistical differences in AAV copy number in the lung.

\section{Discussion}

It has been shown that relative distribution of a substance between the upper, lower respiratory, and gastrointestinal tract in mice following intranasal administration is largely dependent on delivery volume and level of anesthesia [2,3]. Studies have shown that small volumes, specifically those less than $10 \mu \mathrm{l}$, resulted in detection of the administered substance predominantly in the upper respiratory tract, whereas efficient detection in the lower respiratory tract required administration of volumes greater than $50 \mu \mathrm{l}[2,3]$. In addition, intranasal installation was more efficient in anesthetized animals versus awake animals [2], and in animals anesthetized with inhalant anesthetic versus parenteral [3]. Based on these findings, the current study evaluated transduction of the respiratory tract of mice following delivery of $1 \times 10^{11} \mathrm{vg}$ of an AAV serotype 6 vector expressing the human placental alkaline phosphatase (hPLAP) reporter gene in a fixed volume of $80 \mu \mathrm{l}$ to animals that were anesthetized with isoflurane at deep plane in order to contrast and compare four different methods of administration.

The modified-intranasal technique resulted in a similar and consistent distribution of vector to the upper and lower respiratory tract, including all lung lobes, with vector seldom reaching the most distal aspect of the lung. Intratracheal and intubation methods resulted in less consistent vector transduction with a lack of localization to the right middle lung lobe; however, both methods resulted in vector transduction of the distal most part of the lung. The intranasal technique resulted in vector localization primarily within the upper respiratory tract and significantly less vector transduction in the lung compared to the other three methods. These results indicate that the method of vector administration should be chosen based on preferred target site and expertise of the researcher, and that appropriate technique selection may influence experimental outcomes.

The skills and/or amount of training required to perform each technique varied considerably. The 


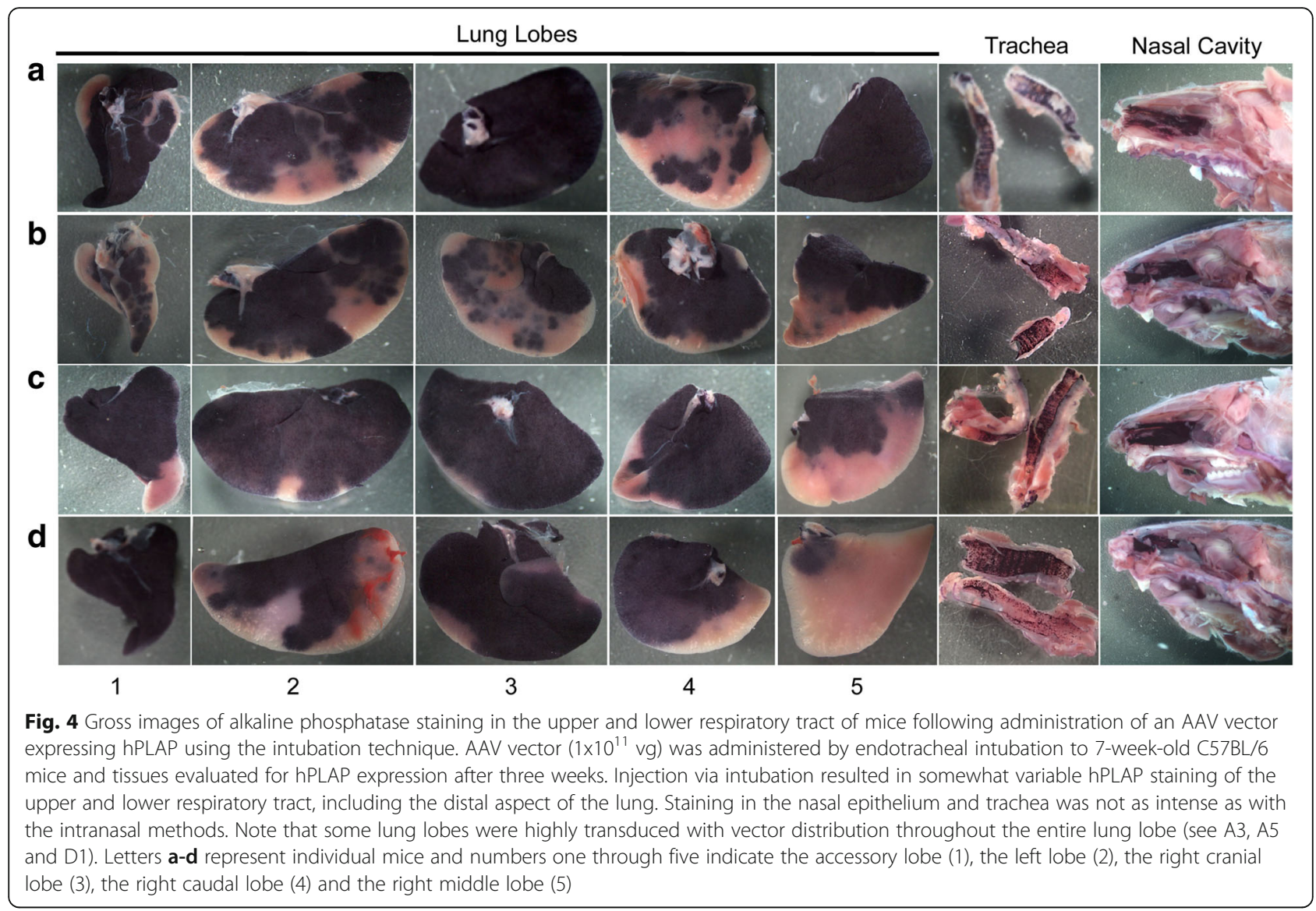

intratracheal injection method required basic surgical skills for closure of the skin incision. The intubation method was inconsistent, likely due to the challenging nature of the technique. Both of these techniques required considerable practice in order to ensure the wellbeing of the animal following recovery, and efficient/ consistent administration of the viral vector to the respiratory tract. In contrast, both intranasal delivery methods required minimal training and efficient and consistent transduction was observed in the respiratory tract following a relatively short time commitment to practicing the modified-intranasal technique. See Table 1 for a summary of the advantages and disadvantages of the four different delivery methods evaluated.

Previous studies have shown that depth of anesthesia can influence the amount of administered substance lost to the gastrointestinal tract $[2,3]$. For both the intranasal and the modified-intranasal technique, the mouse was briefly removed from the source of isoflurane anesthesia prior to vector administration. Therefore, quick recovery and a light plane of anesthesia may have resulted in an increased loss of vector to the gastrointestinal tract. According to our criteria, which were established based on previous experiments, swallowing was documented to have occurred if liquid was found pooling in the mouth of the mouse immediately following vector administration or if bubbles of liquid were released from the mouth during or immediately following vector administration. Unfortunately, because we did not harvest the esophagus and stomach from the animals used in this study, we cannot substantiate these observations. Nevertheless, pooling of liquid in the mouth and production of bubbles was commonly observed with the intranasal delivery method and may have contributed to significantly less vector particles being delivered to the lungs. However, modifying this technique such that the mouth was covered and the animal maintained upright at a $45^{\circ}$ $\mathrm{C}$ angle during vector administration resulted in no apparent loss of vector into the gastrointestinal tract.

Repeat-administration using the intratracheal injection technique may be challenging. The intratracheal injection technique requires a skin incision, which will begin to heal immediately following closure. If readministration is required within a relatively short timeframe, the same incision site could be re-opened since healing would be minimal. Greater than seven days following closure will result in significant fibrosis of the incision site, which may make re-incision challenging. The second incision may need to be made slightly lateral to the first; however, this could impede proper visualization 


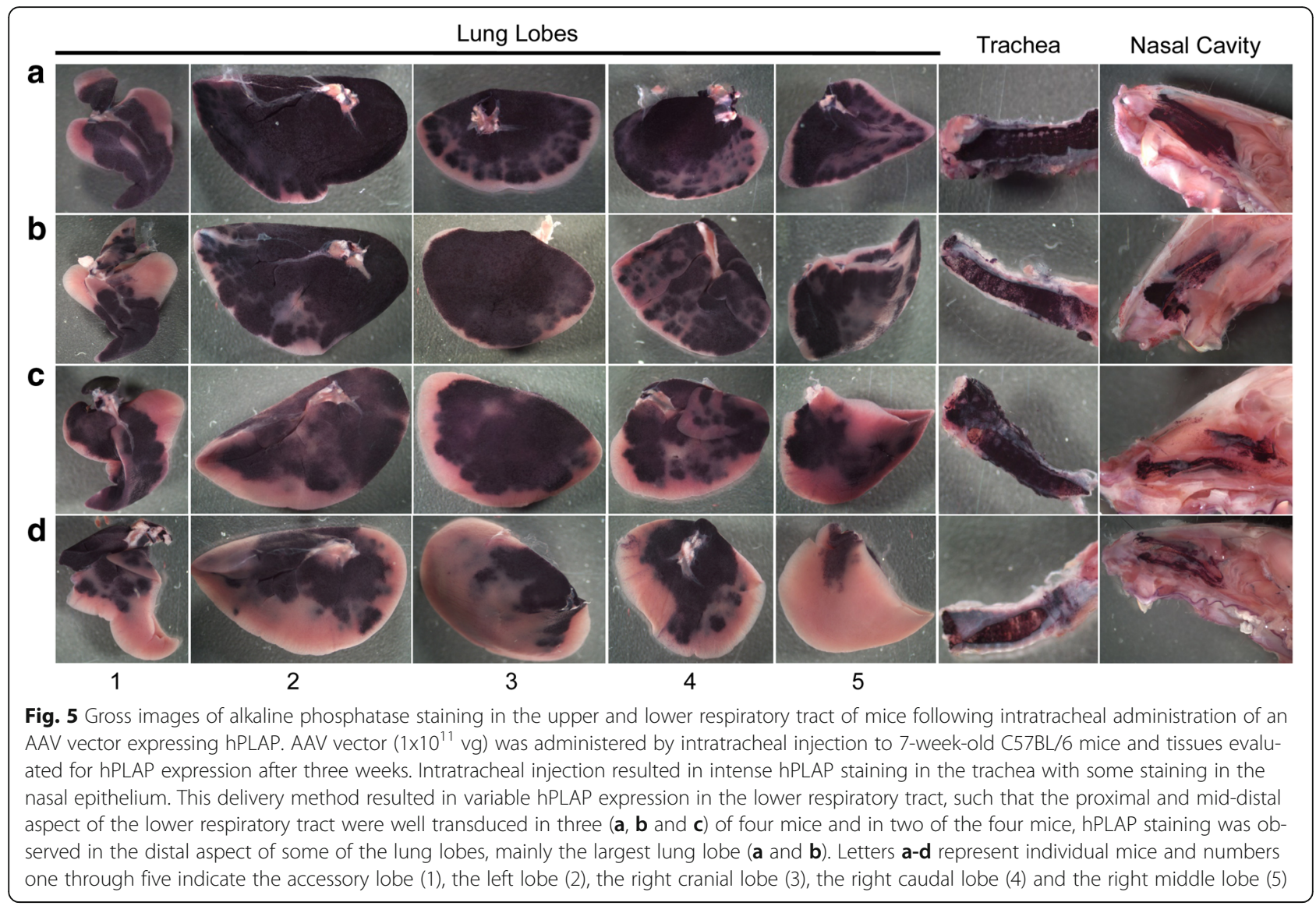

of the trachea. For this reason, if repeat administration is required, an alternate method of administration is recommended.

Additional studies are required to evaluate the extent and character of inflammation resulting from each technique. Control mice received saline in order to evaluate the effects of each technique at the tissue level. No changes consistent with trauma, inflammation or repair were observed (data not shown). However, histopathology at several time points immediately following each technique would need to be evaluated in order to properly characterize whether tissue damage or local inflammation resulted. The intratracheal injection technique likely resulted in the most inflammation locally, at the site of incision; however, evaluation of an acute immune response may be confounded by a local immune response at the site of the incision. When performing the intubation technique, considerable swelling of the tongue and throat may occur if repeated attempts at insertion of the catheter are required. This is more likely to occur if the individual performing the procedure does not practice on a regular basis. If extensive swelling does occur, the procedure must be halted until the swelling has subsided, usually within 24 to 48 hrs. The local and systemic effects of tissue inflammation should be taken into consideration when selection of method of administration is made.

Lastly, analgesia is required for the intratracheal injection technique. The use of certain analgesics may be contraindicated in a particular study. Examples include opioids in behavioural research [6] and NSAIDs in cancer research [7]. However, this should not supersede the use of analgesics [8]. If this is the case, it is recommended that an alternate mode of administration be chosen or a different class of analgesics be utilized.

\section{Conclusions}

In summary, we evaluated three methods of AAV vector administration to the upper and lower respiratory tract in mice: intranasal, intubation, and intratracheal injection. In addition, we presented a modified-intranasal delivery technique (modified-intranasal). All methods of viral vector administration resulted in transduction of the trachea and nasal epithelium, albeit to a lesser extent with the intubation and intratracheal methods of delivery. Intubation was the only method that resulted in transduction of alveoli in the distal most aspect of the lung. The modified-intranasal technique resulted in the most efficient distribution of vector to the upper and lower respiratory tract with the least amount of inter- 


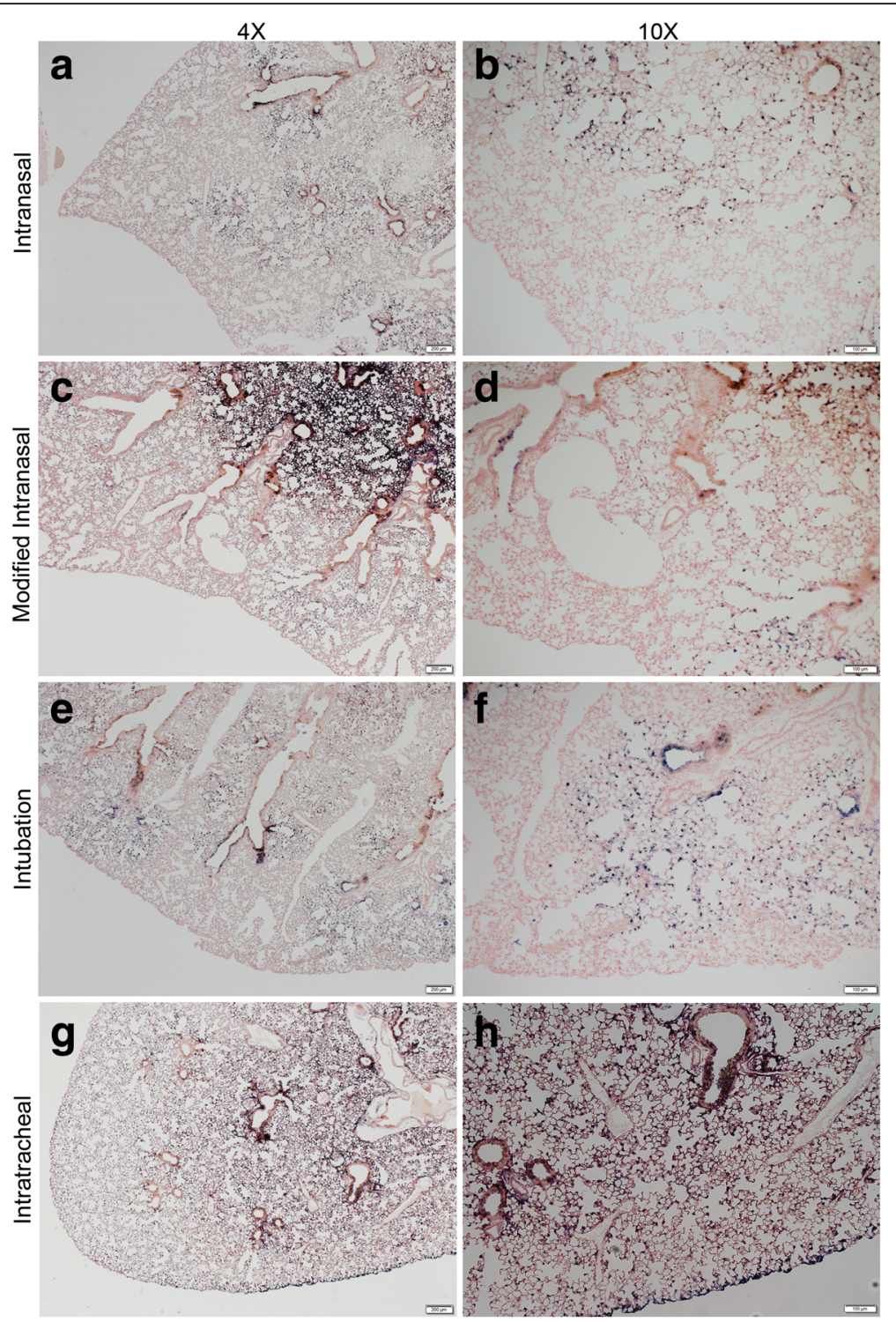

Fig. 6 Histological analysis of alkaline phosphatase staining in the lungs of mice 21 days after delivering $1 \times 10^{11}$ vg of an AAV6 vector expressing hPLAP using four different methods of administration. Viral vectors were administered by the intranasal (a and $\mathbf{b}$ ), modified-intranasal (c and $\mathbf{d}$ ), intubation (e and $\mathbf{f}$ ), or intratracheal injection ( $\mathbf{g}$ and $\mathbf{h}$ ) method. Tissue sections of one representative lung lobe stained for hPLAP expression and counterstained with nuclear fast red are shown at $4 \times(A, C, E, F ;$ scale bar $=200 \mu M)$ and 10x $(B, D, F, H$; scale bar $=100 \mu M)$ magnification

animal variability and requiring the least amount of training. Technique selection may greatly influence experimental outcomes and should be selected based on a variety of factors including time, skill, and desired target site.

\section{Methods}

\section{Animals}

Six-week-old C57BL/6 male mice were purchased from Charles River Laboratories (St Constant, QC). Mice were housed in groups of four and food (Teklad Global 14\% Protein Rodent Maintenance Diet, Indianapolis, USA) and water (tap) were provided ad libitum. Mice were acclimated to the environment for seven days prior to study initiation.

\section{$A A V$ vector production}

Construction of a recombinant AAV vector expressing the reporter gene, human placental alkaline phosphatase (hPLAP), has been described [9]. The packaging plasmid, pDGM6 [10], which encodes the AAV serotype 6 capsid, was kindly provided by Dr. David Russell (University of Washington). AAV vectors and packaging plasmids were propagated in the SURE 2 (Agilent) strain of Escherichia coli. AAV vectors were produced by co-transfection of HEK 293 cells with genome and packaging plasmid as 


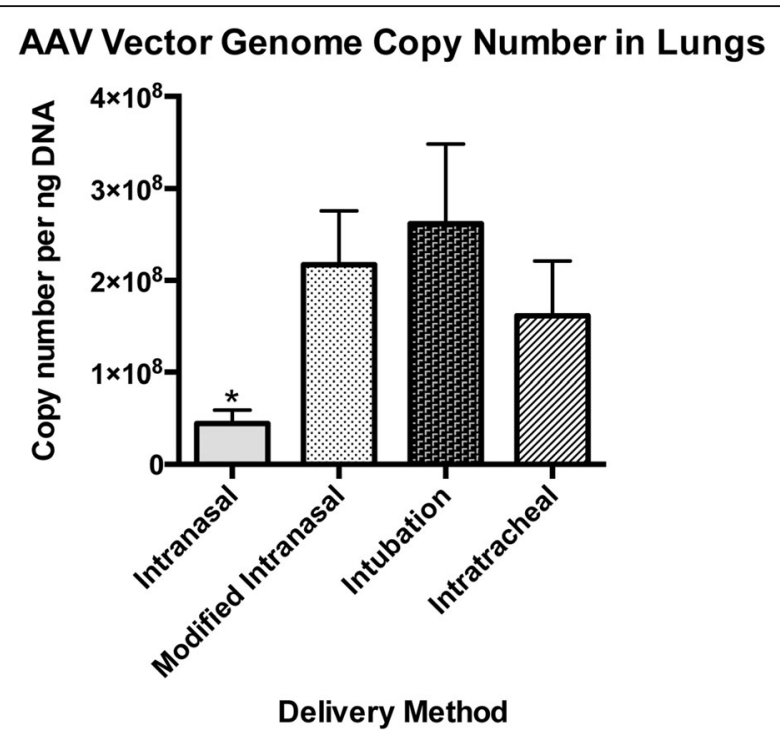

Fig. 7 Vector genome copy numbers in lung tissue. AAV genome copy numbers/ug of genomic DNA were determined 21 days after administration of $1 \times 10^{11} \mathrm{vg}$ of an AAV6 vector expressing hPLAP. Genomic DNA was isolated from lung tissue slices tissues and $100 \mathrm{ng}$ of each was used in triplicate to determine vector genome copies using Taqman qPCR. Levels of significance were determined using one-way analysis of variance followed by Tukey's post-hoc test. The data are shown as mean values \pm SEM. ${ }^{*} P<0.05$ described previously [11]. AAV vector titers were determined by qPCR as described [5]. Each animal was given $1 \times 10^{11} \mathrm{vg}$ of $\mathrm{A}_{\mathrm{JE}} \mathrm{U} 3 \mathrm{CBA}-\mathrm{AP}$, which expresses hPLAP under a composite promoter (Yu et al., manuscript submitted) comprised of the JE enhancer [9] and U3 promoter from Jaagsiekte sheep retrovirus (JSRV) plus the beta-actin promoter, by one of the four delivery methods. Note that the study was performed using $\mathrm{A}_{\mathrm{JE}} \mathrm{U} 3 \mathrm{CBA}-\mathrm{AP}$ vector from one production batch.

\section{Intranasal Technique}

Mice were anesthetized (induced and maintained) individually inside a feline nose cone with $2.5 \%$ isoflurane (Aerrane, Baxter Corp, Mississauga, $\mathrm{ON}$ ) delivered in $\mathrm{O}_{2}$ (1 $\mathrm{L} / \mathrm{min})$. Immediately prior to delivering the vector, the mouse was removed from the nose cone and placed in dorsal recumbency (Fig. 1a). A preliminary volume of $40 \mu \mathrm{l}$ of vector was delivered drop wise over the nares and passively inhaled. The mice were permitted to recover from anesthesia for $10 \mathrm{~min}$ in a recovery cage and the procedure repeated (total of $80 \mu \mathrm{l}$ of vector administered).

\section{Modified-intranasal Technique}

Mice were anesthetized (induced and maintained) with $2.5 \%$ isoflurane in $\mathrm{O}_{2}(1 \mathrm{~L} / \mathrm{min})$ as described for the intranasal technique. Immediately prior to delivering the vector, the mouse was removed from the nose cone and gently restrained by the scruff at a $45^{\circ}$ angle using the

Table 1 Summary of the advantages and disadvantages of the four delivery methods evaluated

\begin{tabular}{|c|c|c|c|}
\hline Technique & Anatomic Target & Advantages & Disadvantages \\
\hline Intranasal & $\begin{array}{l}\text { - Nasal epithelium } \\
\text { - Tracheal epithelium } \\
\text { - Proximal aspect of lung lobes }\end{array}$ & $\begin{array}{l}\text { - Relatively quick } \\
\text { - Little skill/training required } \\
\text { - Can be performed by one person } \\
\text { - Inexpensive } \\
\text { - Best option if wanting to target the } \\
\text { upper respiratory tract } \\
\text { - Can administer multiple doses }\end{array}$ & $\begin{array}{l}\text { - Frequent loss of vector due to swallowing } \\
\text { - Inefficient delivery to the } \\
\text { lower respiratory tract }\end{array}$ \\
\hline Modified intranasal & $\begin{array}{l}\text { - Nasal epithelium } \\
\text { - Tracheal epithelium } \\
\text { - Proximal to middle aspect of } \\
\text { lung lobes }\end{array}$ & $\begin{array}{l}\text { - Relatively quick } \\
\text { - Little skill/training required } \\
\text { - Inexpensive } \\
\text { - Least variable delivery method } \\
\text { - Can administer multiple doses }\end{array}$ & $\begin{array}{l}\text { - Requires two people } \\
\text { - Does not reach the distal aspect of lung lobes }\end{array}$ \\
\hline Intubation & $\begin{array}{l}\text { - Nasal epithelium } \\
\text { - Tracheal epithelium } \\
\text { - Proximal, middle and distal } \\
\text { aspect of lung lobes }\end{array}$ & $\begin{array}{l}\text { - Method that most } \\
\text { consistently reaches the distal aspect of } \\
\text { the lung lobes } \\
\text { - Can be performed by one person }\end{array}$ & $\begin{array}{l}\text { - Technically challenging } \\
\text { - Requires more equipment than the other methods } \\
\text { - Variable } \\
\text { - Time consuming } \\
\text { - Possibility of losing } \\
\text { vector if esophagus is penetrated } \\
\text { - Excessive swelling prevents repeated attempts at } \\
\text { intubation }\end{array}$ \\
\hline Intratracheal & $\begin{array}{l}\text { - Nasal epithelium } \\
\text { - Tracheal epithelium } \\
\text { - Proximal to middle } \\
\text { aspect of lung lobes } \\
\text { - Possible to target distal } \\
\text { aspect of lung lobes }\end{array}$ & $\begin{array}{l}\text { - Primarily targets the lower } \\
\text { respiratory tract } \\
\text { - Limited delivery to the nasal epithelium } \\
\text { - Can be performed by one person, but } \\
\text { is easier with two people }\end{array}$ & $\begin{array}{l}\text { - Technically challenging } \\
\text { - Requires surgical skills } \\
\text { - Variable } \\
\text { - Invasive } \\
\text { - Requires analgesic } \\
\text { - Re-administration not advised if done more than } \\
5 \text { days after initial administration }\end{array}$ \\
\hline
\end{tabular}


right hand and the mouth gently pinched shut using the thumb and forefinger of the left hand so as to prevent mouth breathing. Once the mouse was restrained and the mouth properly covered, a second individual delivered a preliminary volume of $40 \mu \mathrm{l}$ of vector drop wise over the nares and the drops passively inhaled (Fig. 1b). The mouth was uncovered once all of the liquid was inhaled. The mouse was maintained in this position for approximately $30 \mathrm{~s}$ after vector delivery to allow for passive distribution of vector into the lower respiratory tract. The mice were permitted to recover for approximately $10 \mathrm{~min}$ in a recovery cage and the procedure repeated (total of $80 \mu \mathrm{l}$ of vector administered).

\section{Intubation Technique}

Mice were anesthetized (induced and maintained) with $2.5 \%$ isoflurane in $\mathrm{O}_{2}(1 \mathrm{~L} / \mathrm{min})$ and positioned in dorsal recumbency on a metal board oriented at a $45^{\circ}$ angle with the rostral end towards the user (Fig. 1c). The upper incisor teeth were hooked under an elastic band attached to the metal board. Mice were exposed to isoflurane throughout the procedure via polyurethane connector tubing placed over the nares. A flexible, highintensity light source (e.g. Fiber-Lite ${ }^{\circ}$ high intensity fiber optic flexible illuminator, Dolan-Jenner Industries, MA United States) was positioned $1 \mathrm{~cm}$ from the ventral aspect of the sternum. The light shines rostrally through the trachea, allowing for differentiation of the lumen of the trachea from that of the esophagus. A set of serrated blunt nose curved $\left(20^{\circ}\right.$ angle) stainless steep forceps (Fine Scientific Tools, item\# 11051-10) were used to gently extend the mouse's tongue out and to the side of the mouth allowing for direct visualization of the epiglottis and larynx. The tongue was held in this position throughout the procedure. Next, blunt stainless steel forceps bent at a $90^{\circ}$ angle (Fine Scientific Tools, item\# 11052-10) were inserted under the mouse's tongue and carefully lifted dorsally to allow for greater visualization and access to the trachea. At this point, small adjustments were made to visualize the epiglottis. Using the dominant hand, a 22-gauge catheter was placed between the index and middle finger and stabilized with the thumb in order to direct the catheter through the mouth and larynx into the trachea, stopping just before the bifurcation of the trachea (Fig. 1c). The metal stylette was quickly removed and the polyurethane tubing attached to the catheter opening to allow for continued delivery of isoflurane. Once properly intubated, the polyurethane tube was briefly removed from the end of the catheter, and using a P200 pipette, $80 \mu \mathrm{l}$ of vector was carefully injected into the catheter as the mouse inhaled. Note that approximately $100 \mu \mathrm{l}$ of air was drawn up into the pipette tip prior to drawing up $80 \mu \mathrm{l}$ of vector so as to prevent loss of vector and to promote delivery of the vector deep into the lungs. The mouse was left in this position for $5 \mathrm{~min}$ to allow for passive distribution of vector into the lower respiratory tract.

\section{Intratracheal Injection Technique}

Mice were anesthetized (induced and maintained) with $2.5 \%$ isoflurane in $\mathrm{O}_{2}(1 \mathrm{~L} / \mathrm{min})$ and positioned in dorsal recumbency on a metal board oriented at a $45^{\circ}$ angle with the rostral end away from the user. A $0.5 \mathrm{~cm}$ incision was made through the skin, directly cranial to the manubrium. Subcutaneous tissue was carefully dissected away and tissue forceps were used to gently lateralize salivary glands to visualize the trachea (Fig. 1d). Note that applying gentle downward pressure with forceps onto either side of the trachea allows for better visualization of the tracheal rings, which is essential to confirming the appropriate injection location. Using a 29-gauge tuberculin syringe (BD, New Jersey USA) at a $30-45^{\circ}$ angle, $80 \mu \mathrm{l}$ of virus was injected into the trachea. The skin was then closed with 5-0 poliglecaprone 25 (Monocryl, Ethicon, Sommerville, USA) in a single cruciate pattern.

\section{Tissue Preparation and Staining}

Mice were euthanized three weeks following vector administration. The lung, trachea, and nasal cavity were harvested and fixed in $2 \%$ paraformaldehyde for 24 to $48 \mathrm{hr}$ at room temperature. After washing three times in phosphate buffered saline (PBS) to remove the fixative, tissues were transferred to $65^{\circ} \mathrm{C}$ PBS, incubated for $1 \mathrm{hr}$ in a water bath at $65{ }^{\circ} \mathrm{C}$ to inactivate endogenous alkaline phosphatase and then stained for hPLAP expression as previously described [9]. After gross images were obtained, tissues were paraffin embedded and sectioned at $4 \mu \mathrm{m}$.

\section{Genomic DNA extraction and AAV copy number analysis}

Five serial sections of 8-micrometer thickness each per sample were taken from each animal using a standard microtome with disposable DNA-RNA free blades. Tissue slices were obtained after trimming to remove tissue exposed to oxygen and to ensure that all five lung lobes were contained within the same plane in the tissue slices. The five tissue slices were placed into a sterile $1.5 \mathrm{ml}$ microfuge tube and genomic DNA was isolated using the QIAamp DNA FFPE Tissue Kit according to the manufacturer's instructions. DNA was quantified using the Qubit fluorometer and PicoGreen quantification reagents (Invitrogen). Vector genome copy number per ng of genomic DNA was determined by Taqman qPCR starting from $100 \mathrm{ng}$ of mouse gDNA by amplifying the AAV ITRs and mouse beta actin $(A c t b)$ as a mouse housekeeping gene. The absolute amount of each gene was obtained by referring to a standard curve 
consisting of a 10-fold serial dilution of a plasmid containing two copies of the AAV ITR and one copy of the $A c t b$ gene sequence (six concentrations, ranging from $10-10^{-6}$ copies). Real-time PCR assays were conducted using a StepOnePlus Real-Time PCR system from Thermo Fisher Scientific Inc. Ten $\mu \mathrm{l}$ reactions containing $5 \mu \mathrm{l} 2 \times$ Taqman Master Mix (PerFeCta FastMix II from Quanta BioScience (Cat. No. 95119-250), 1ul of PCR primer/probes (final concentration of primer was $500 \mathrm{nM}$ and probe was $250 \mathrm{nM}$ ) and $4 \mu \mathrm{l}$ of plasmid DNA in serial dilutions. The run conditions were as follows: $30 \mathrm{~s}$ at $95^{\circ} \mathrm{C}$ polymerase activation step, followed by 35 to 40 cycles of a two-step qPCR $\left(3 \mathrm{~s}\right.$ of $95{ }^{\circ} \mathrm{C}$ denaturation, $30 \mathrm{~s}$ of $60{ }^{\circ} \mathrm{C}$ combined annealing/extension). Primers and probes were purchased from IDT and used as follows: AAV ITR, 5'-GGAACCCCTAGTGATGGAGTT-3' (forward), 5'-CGGCCTCAGTGAGCGA-3' (reverse), 56-FAM/CACTCCCTC/Zen/T CTGCGCGC TCG/3IABkFQ (probe) [5]; and mouse Actb PrimeTime Std $^{\circ}$ qPCR Assay from IDT (Mm.PT.39a.22214843.g).

\section{Statistical analysis}

Data were analyzed with Graphpad Prism version 5 (Graphpad Software) and expressed as mean \pm standard error of the mean. Statistical differences between mean values were tested using one-way ANOVA followed by the Tukey's post-hoc test. Differences between values were considered to be significant with: ${ }^{*} P<0.05$.

\section{Additional files}

Additional file 1: Figure S1. Histological analysis of alkaline phosphatase staining in the lungs of mice 21 days after delivering $1 \times 10^{11}$ $\mathrm{vg}$ of an AAV6 vector expressing hPLAP by the intranasal method of administration. Tissue sections of all five lung lobes were stained for hPLAP expression and counterstained with nuclear fast red and representative images are shown. Images were taken at $1.5 \mathrm{x}$ magnification. Scale bar $=1 \mathrm{~mm}$. (TIF $6183 \mathrm{~kb}$ )

Additional file 2: Figure S2. Histological analysis of alkaline phosphatase staining in the lungs of mice 21 days after delivering $1 \times 10^{11}$ $\mathrm{vg}$ of an AAV6 vector expressing hPLAP by the modified intranasal method of administration. Tissue sections of all five lung lobes were stained for hPLAP expression and counterstained with nuclear fast red and representative images are shown. Images were taken at $1.5 \mathrm{x}$ magnification. Scale bar $=1 \mathrm{~mm}$. (TIF $8321 \mathrm{~kb}$ )

Additional file 3: Figure S3. Histological analysis of alkaline phosphatase staining in the lungs of mice 21 days after delivering $1 \times 10^{11}$ vg of an AAV6 vector expressing hPLAP by the intubation method of administration. Tissue sections of all five lung lobes were stained for hPLAP expression and counterstained with nuclear fast red and representative images are shown. Images were taken at $1.5 \mathrm{x}$ magnification. Scale bar $=1 \mathrm{~mm}$. (TIF $7519 \mathrm{~kb}$ )

Additional file 4: Figure S4. Histological analysis of alkaline phosphatase staining in the lungs of mice 21 days after delivering $1 \times 10^{11}$ vg of an AAV6 vector expressing hPLAP by the intratracheal injection method of administration. Tissue sections of all five lung lobes were stained for hPLAP expression and counterstained with nuclear fast red and representative images are shown. Images were taken at $1.5 \mathrm{x}$ magnification. Scale bar $=1 \mathrm{~mm}$. (TIF $7928 \mathrm{~kb}$ )
Additional file 5: Figure S5. Histological analysis of alkaline phosphatase staining in the lungs and trachea of mice 21 days after delivering $1 \times 10^{11} \mathrm{vg}$ of an AAV6 vector expressing hPLAP using four different methods of administration. Viral vectors were administered by the intranasal ( $A$ and $E)$, modified-intranasal ( $B$ and $F$ ), intubation ( $C$ and $\mathrm{G}$ ), or intratracheal injection ( $\mathrm{D}$ and $\mathrm{H}$ ) method. Tissue sections of one representative lung lobe stained for hPLAP expression and counterstained with nuclear fast red are shown at 20x magnification (A-D; scale $\mathrm{bar}=50 \mu \mathrm{M})$. Tissue sections of one representative trachea stained for hPLAP expression and counterstained with nuclear fast red are shown at 20x magnification ( $\mathrm{E}-\mathrm{H}$; scale bar $=50 \mu \mathrm{M})$. (TIF $17789 \mathrm{~kb})$

\section{Acknowledgements}

We would like to acknowledge the technical assistance of Ms. Betty-Anne McBey and staff at the Animal Isolation Unit at the University of Guelph. LAS was the recipient of an Alexander Graham Bell NSERC PhD Scholarship. JCI and LPV were the recipients of an Ontario Graduate Scholarship (OGS) and an Ontario Veterinary College Fellowship.

\section{Funding}

Funding for this study was provided by Cystic Fibrosis Canada and the Ontario Lung Association. CF Canada and Ontario Lung Association had no role in the study design, collection, analysis or interpretation of the data, writing the manuscript, or the decision to submit the paper for publication.

\section{Availability of data and material}

The datasets during and/or analyzed during the current study are available from the corresponding author upon request.

\section{Authors' contributions}

SKW conceived of and designed the study. LAS, JCI, DLY, JGDJ, LPV, GAW, and SKW performed the study. JCI, LAS and SKW drafted/revised the manuscript. All authors read and approved the final manuscript.

\section{Competing interests}

The authors declare that they have no competing interests.

\section{Consent for publication}

Not applicable

\section{Ethics approval}

The University of Guelph Animal Care Committee approved the animal use protocol, and the facility and procedures are in compliance with the Animals for Research Act of Ontario and the guidelines of the Canadian Council on Animal Care.

\section{Publisher's Note}

Springer Nature remains neutral with regard to jurisdictional claims in published maps and institutional affiliations.

Received: 10 January 2017 Accepted: 8 May 2017

Published online: 15 May 2017

\section{References}

1. Keeler AM, Flotte TR. Cell and gene therapy for genetic diseases: inherited disorders affecting the lung and those mimicking sudden infant death syndrome. Hum Gene Ther. 2012;23(6):548-56.

2. Southam DS, Dolovich M, O'Byrne PM, Inman MD. Distribution of intranasal instillations in mice: effects of volume, time, body position, and anesthesia. Am J Physiol Lung Cell Mol Physiol. 2002;282(4):L833-839.

3. Miller MA, Stabenow JM, Parvathareddy J, Wodowski AJ, Fabrizio TP, Bina $X R$, Zalduondo L, Bina JE. Visualization of murine intranasal dosing efficiency using luminescent Francisella tularensis: effect of instillation volume and form of anesthesia. PLoS One. 2012;7(2):e31359.

4. Halbert C, Lam S, Miller A. High-efficiency promoter-dependent transduction by adeno-associated virus type 6 vectors in mouse lung. Hum Gene Ther. 2007;18(4):344-54.

5. Aurnhammer C, Haase M, Muether N, Hausl M, Rauschhuber C, Huber I, Nitschko $H$, Busch U, Sing A, Ehrhardt A, et al. Universal real-time PCR for the detection and quantification of adeno-associated virus serotype 2- 
derived inverted terminal repeat sequences. Hum Gene Ther Methods. 2012;23(1):18-28

6. Berrocoso E, Ikeda K, Sora I, Uhl GR, Sánchez-Blázquez P, Mico JA. Active behaviours produced by antidepressants and opioids in the mouse tail suspension test. Int J Neuropsychopharmacol. 2013;16(1):151-62.

7. Thun MJ, Henley SJ, Patrono C. Nonsteroidal anti-inflammatory drugs as anticancer agents: mechanistic, pharmacologic, and clinical issues. J Natl Cancer Inst. 2002;94(4):252-66.

8. National Research Council (US) Committee for the Update of the Guide for the Care and Use of Laboratory Animals. Guide for the Care and Use of Laboratory Animals. In., 8th edn. Washington, DC: National Academies Press (US); 2011.

9. Yu DL, Linnerth-Petrik NM, Halbert CL, Walsh SR, Miller AD, Wootton SK Jaagsiekte sheep retrovirus and enzootic nasal tumor virus promoters drive gene expression in all airway epithelial cells of mice but only induce tumors in the alveolar region of the lungs. J Virol. 2011;85(15):7535-45.

10. Gregorevic P, Blankinship MJ, Allen JM, Crawford RW, Meuse L, Miller DG, Russell DW, Chamberlain JS. Systemic delivery of genes to striated muscles using adeno-associated viral vectors. Nat Med. 2004;10(8):828-34.

11. Halbert C, Allen J, Miller A. Efficient mouse airway transduction following recombination between $A A V$ vectors carrying parts of a larger gene. Nat Biotechnol. 2002;20(7):697-701.

Submit your next manuscript to BioMed Central and we will help you at every step:

- We accept pre-submission inquiries

- Our selector tool helps you to find the most relevant journal

- We provide round the clock customer support

- Convenient online submission

- Thorough peer review

- Inclusion in PubMed and all major indexing services

- Maximum visibility for your research

Submit your manuscript at www.biomedcentral.com/submit
Biomed Central 